\title{
Mass Discrimination in High-Mass MALDI-MS
}

\section{Journal Article}

\section{Author(s):}

Weidmann, Simon; Mikutis, Gediminas; Barylyuk, Konstantin; Zenobi, Renato

Publication date:

2013

Permanent link:

https://doi.org/10.3929/ethz-b-000071138

Rights / license:

In Copyright - Non-Commercial Use Permitted

\section{Originally published in:}

Journal of the American Society for Mass Spectrometry 24(9), https://doi.org/10.1007/s13361-013-0686-x 


\title{
Mass Discrimination in High-Mass MALDI-MS
}

\author{
Simon Weidmann, Gediminas Mikutis, Konstantin Barylyuk, Renato Zenobi
}

Department of Chemistry and Applied Biosciences, ETH (Swiss Federal Institute of Technology) Zürich, CH-8093 Zürich, Switzerland

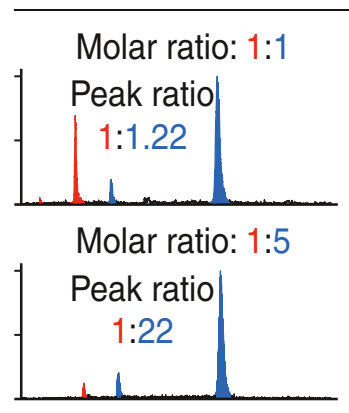

\begin{abstract}
In high-mass matrix-assisted laser desorption/ionization mass spectrometry (MALDI-MS), the accessible $\mathrm{m} / \mathrm{z}$ range is limited by the detector used. Therefore, special high-mass detectors based on ion conversion dynodes (ICDs) have been developed. Recently, we have found that mass bias may exist when such ICD detectors are used [Weidmann et al., Anal. Chem. 85(6), 3425-3432 (2013)]. In this contribution, the mass-dependent response of an ICD detector was systematically studied, the response factors for proteins with molecular weights from 35.9 to $129.9 \mathrm{kDa}$ were determined, and the reasons for mass bias were identified. Compared with commonly employed microchannel plate detectors, we found that the mass discrimination is less pronounced,
\end{abstract} although ions with higher masses are weakly favored when using an ICD detector. The relative response was found to depend on the laser power used for MALDI; low-mass ions are discriminated against with higher laser power. The effect of mutual ion suppression in dependence of the proteins used and their molar ratio is shown. Mixtures consisting of protein oligomers that only differ in mass show less mass discrimination than mixtures consisting of different proteins with similar masses. Furthermore, mass discrimination increases for molar ratios far from 1. Finally, we present clear guidelines that help to choose the experimental parameters such that the response measured matches the actual molar fraction as closely as possible.

Key words: Relative response factor, Mass discrimination, MALDI-TOF-MS, High-mass protein analysis, lon conversion dynodes

Received: 25 April 2013/Revised: 27 May 2013/Accepted: 28 May 2013/Published online: 9 July 2013

\section{Introduction}

A nalysis of complex sample mixtures is one of the most difficult tasks in analytical science, since usually not all constituents of the mixture are equally well detected. Matrixassisted laser desorption/ionization mass spectrometry (MALDI-MS) proved to be a fast and sensitive technique for analysis of mixtures consisting of proteins [1] or synthetic polymers [2] already in the early days after its invention. When analyzing such complex mixtures, it is important to keep the analytes intact, thus soft ionization techniques must be applied. An alternative method that is often applied is electrospray ionization (ESI). MALDI has, however, a major advantage over ESI for the analysis of mixtures, which is the production of predominantly singly charged ions. This greatly facilitates the interpretation of spectra, since only few overlapping signals are generated. Furthermore, the measured mass-to-charge ratios $(\mathrm{m} / \mathrm{z})$ give

Correspondence to: Renato Zenobi; e-mail: zenobi@org.chem.ethz.ch direct information on the masses of all analytes contained in a mixture.

MALDI-MS has become a routine tool for analysis of complex synthetic polymer samples. Polymers are almost never synthesized with a well-defined length and polymerization degree, but normally show an oligomer distribution, thus yielding complex mixtures. With MALDI-MS it is possible to determine, for example, molecular weight distributions and structural features such as end groups [3, 4].

When characterizing synthetic polymers, it is crucial that the masses and the degree of polymerization of the samples are determined correctly. Studies of mass discrimination in MALDI-MS were performed in the context of polymer analysis, and considered a broad range of parameters [5-9]. However, it has been found that the mass distribution depends to a great extent on the parameters chosen for sample preparation, such as solvent or type and concentration of the cationization agent and matrix used, and on parameters for desorption/ionization (e.g., the laser power). Analysis of DNA is another case where rather heterogeneous sample mixtures are investigated using MALDI-MS and the sequence can be 
determined [10-12]. However, it has been shown that in MALDI-MS, certain constituents of such oligonucleotide mixtures are discriminated against because of different factors such as detector efficiency and ion suppression [13]. In an extreme case, a sample can be suppressed completely, thus render the sequence analysis challenging, if not impossible.

When investigating protein mixtures, it is not only interesting to identify the constituents but also their interaction with each other and other biomolecules. Unfortunately, in MALDI these noncovalent interactions are easily disrupted. Therefore, some techniques to preserve these interactions were developed to allow the determination of the stoichiometric composition of complexes [14, 15].

Furthermore, it has been shown in a recent publication that the stabilization of noncovalent interactions using chemical crosslinking also allows the determination of dissociation constants $\left(\mathrm{K}_{\mathrm{D}}\right)$ of protein-ligand interactions [16]. To measure the $K_{D}$, the protein and the ligand are incubated and cross-linked. After MALDI-MS analysis, the ratio of non-cross-linked and cross-linked protein-ligand complex is calculated and the $\mathrm{K}_{\mathrm{D}}$ determined. The precision of $\mathrm{K}_{\mathrm{D}}$ determination by this method relies on the assumption of an identical ion response over the complete $\mathrm{m} / \mathrm{z}$ range. Factors influencing the response are, for example, the ionization process, the ion transmission of the instrument, and the detection of ions [17].

Owing to the low charge states generated in MALDI, the $\mathrm{m} / \mathrm{z}$ ratio might become rather high. A precise determination of the sample's mass becomes difficult since no suitable, commercially available calibrants exist. In a recent work by our group, a new, modular calibrant has been presented [18]. These calibrants consist of several covalently linked copies of the same protein and, therefore, the samples properties such as the relative amino acid composition are identical. Obviously, because of the different number of protein repeats, the samples differ in mass. Evaluating this calibrant showed an unequal response of the MALDI-MS used for equimolar mixtures of calibrants. Such a mass-dependent response is called mass discrimination $[17,19]$. Similar discrimination effects were observed in the past when complex mixtures consisting of DNA [13], proteins [19], or polymers [6-9] were investigated.

As mentioned above, one of the possible reasons for mass discrimination is a different detection efficiency of the ions. A commonly used detector in time-of-flight (TOF) MS is the microchannel plate (MCP) detector. This detector consists of an array of tiny tubes in which the impinging ions are converted into secondary electrons, which are further multiplied by an electron cascade to amplify the signal [20]. Unfortunately, MCPs suffer from a decreased sensitivity towards ions with high mass, primarily due to the low ion-to-electron conversion yield of ions with low velocities [21-23] and secondly due to saturation of the microchannels upon impact of low-mass (e.g., matrix) ions [24]. The effects of this difference in sensitivity on the obtained signal have been studied by Farmer and Caprioli using cross-linked oligomeric protein complexes [19]. In this study, the suppression was mainly attributed to the detector used and correction factors to compensate for the reduced detection efficiency were determined.

To overcome the problem of low detection efficiency of high-mass samples, other detector technologies, for example, cryogenic detectors $[25,26]$, mechanical nanomembrane detectors [27], or ion conversion dynode (ICD) detectors [28], were developed. To our knowledge, only ICD detectors are currently commercially available.

ICD detectors rely on an ion-to-secondary ion conversion. The yield of secondary ions created at the ICD increases with the mass of the impinging ion, similarly to secondary ion mass spectrometry $[29,30]$. This leads to an enhanced sensitivity towards high-mass ions. At this time, it is unknown if measurements performed by ICD detectors suffer from any kind of mass discrimination or if the results reflect the sample composition correctly.

In order to investigate whether mass discrimination in highmass MALDI-MS exists and to identify the underlying reasons, an experiment to determine the relative response of protein samples was designed. Since the ionization efficiency in MALDI depends to a great extent on the chemical properties of the sample, molecules differing only in mass were chosen. The recently published concatenated polyproteins based on maltodextrinbinding protein (MBP) as subunit were chosen as the model system [18]. These biomolecules consist of several covalently linked copies of the same protein. Therefore, the ionization properties are expected to be similar. The main difference between the two species chosen here (MBP and $\left.\mathrm{MBP}_{3}\right)$ is the mass of 43 and $130 \mathrm{kDa}$, respectively.

Not only mass can be of importance but also the nature of the sample itself: in a very recent study by Chen et al., it was documented that the two transmembrane subunits of a heterotetrameric complex are much harder to detect using high-mass MALDI-MS than the two soluble subunits [31]. To study differences in sample properties, a designed ankyrin repeat protein (DARPin) with a similar mass than MBP was chosen, which has a different amino acid composition. While in the MBP polyprotein units of $43 \mathrm{kDa}$ are connected, DARPins are modular proteins with much smaller units. Between an Nterminal and a C-terminal capping repeat, several, here 8 or 10 , ankyrin repeats (AR) of 33 amino acids are inserted. In the present work, all internal repeats have the same sequence [32]. DARPins can thus be used to create a finely graded ladder of proteins with defined molecular weights (dependent on the number of repeats). In this study, all internal repeats had the same sequence, although this can be varied over a wide range [32-34].

The samples proposed here are better suited for determining response factors than the cross-linked protein samples used by Farmer and Caprioli because they are (1) recombinantly expressed in Escherichia coli and therefore do not contain post-translational modifications, and (2) are covalently linked, thus no cross-linking is necessary. Moreover, the cross-linker used in the previous study was glutaraldehyde, which leads to extensive peak broadening [19]. Since later on the peak integrals were compared, this peak broadening is a potential source of error. 
Up to now, analysis of complex protein mixtures with MALDI-MS only allowed the detection and identification of these proteins. No answer could be given on the amounts and molar ratios of the proteins present. With the procedure presented in this work, it is possible to identify factors that lead to mass discrimination in high-mass MALDI-MS. Thus, it becomes possible to set up experiments in a way that minimizes mass discrimination. Once the relative response for the mixture of interest is known, it becomes possible to take care of the discrimination and eventually correct for it. Therefore, information about the relative response of the sample under investigation can improve, e.g., the mass distribution of complex polymer samples investigated) by high-mass MALDI-MS.

\section{Experimental}

\section{Materials}

$\mathrm{MBP}$ and $\mathrm{MBP}_{3}$ were expressed and purified as described before [18]. The DARPins used (N8C and N10C), constructed analogously to the shorter ones described before [32] were constructed and purified by Rastislav Tamaskovic, generously provided by the Plückthun Lab (University of Zürich, Switzerland) and used as received. Sinapinic acid (SA), acetonitrile (ACN), and phosphate buffered saline (PBS) tablets ( $\mathrm{pH}$ 7.4) were purchased from Sigma-Aldrich (Buchs, Switzerland), trifluoroacetic acid (TFA) from Acros Organics (Geel, Belgium), and sodium chloride $(\mathrm{NaCl})$ from J. T. Baker (Deventer, The Netherlands). All chemicals were of the highest purity available and were used without further purification. Water with a resistivity of $18.2 \mathrm{M} \Omega \cdot \mathrm{cm}$ was prepared using a NANOpure Diamond water purification system (Barnstead Int., Dubuque, IA, USA).

\section{Sample Preparation}

The concentrations of the samples were determined by measuring the absorbance at $280 \mathrm{~nm}$ using an UV/Vis spectrophotometer (GENESYS 10S UV-Vis; Thermo Scientific, Madison, WI, USA). The extinction coefficients (ع) were calculated based on the primary sequence of the proteins using the ExPASy Proteomics tools (see Table 1). Prior to analysis, the proteins were diluted with water and PBS

Table 1. The Theoretical Molecular Weights (MW), Extinction Coefficients $(\varepsilon)$, and Isoelectric Points (pI) of the Substances Under Investigation were Calculated Using ExPASy Proteomics Tools. The Detection Limits (LoD) Were Determined by Dilution of the Samples to a Concentration where the Measured Signal-to-Noise Ratio was Still Above 3:1

\begin{tabular}{lcccc}
\hline Name & MW $(\mathrm{kDa})$ & $\varepsilon\left(\mathrm{M}^{-1} \mathrm{~cm}^{-1}\right)$ & $\mathrm{pI}$ & LoD (fmol) \\
\hline MBP & 43.2 & 66350 & 5.48 & 25 \\
MBP $_{3}$ & 129.9 & 200540 & 5.13 & 13 \\
N8C & 35.9 & 11920 & 5.49 & 25 \\
N10C & 42.9 & 14900 & 5.48 & 25 \\
\hline
\end{tabular}

to the required concentrations in such a way that the buffer concentration was kept constant. The total protein concentration was kept constant at $0.8 \mu \mathrm{M}$ unless otherwise noted.

The matrix solution was prepared by dissolving SA at a concentration of $10 \mathrm{mg} / \mathrm{mL}$ in ACN/water/TFA (49.5:49.5:1; vol:vol:vol). Matrix and sample were prepared in a 1:1 ratio (vol:vol) and thoroughly mixed. Per spot, $0.5 \mu \mathrm{L}$ aliquots of this mixture were spotted onto a stainless steel MALDI plate and allowed to crystallize under ambient conditions.

\section{Mass Spectrometry}

The experiments were performed on a commercial MALDIMS instrument (MALDI TOF/TOF 4800 Plus; AB SCIEX, Darmstadt, Germany) equipped with a high-mass ICD-detector (HM2tuvo; CovalX, Zürich, Switzerland). The MS was operated in linear mode with an effective flight path of $1.7 \mathrm{~m}$. The laser used for desorption/ionization is a frequency-tripled $\mathrm{Nd}$ :YAG laser $(355 \mathrm{~nm})$, and the laser power was chosen to be just above the threshold for ion formation unless otherwise declared; 250 laser shots were recorded automatically at random spot positions and averaged to create a mass spectrum. The spectra were recorded using the manufacturer's software (4000 Series Explorer V.3.5.3) and exported as ASCII files for further processing.

\section{Data Processing}

The exported data were smoothed and baseline-corrected using the provided Savitzky-Golay algorithm of Origin 8.6.0 (OriginLab Corp., Northampton, MA, USA). The signal intensity and resolution (full width at half maximum [FWHM] definition) for each peak were checked and the peak integrals calculated.

To calculate the relative response factors $\left(\mathrm{R}_{\text {rel }}\right)$ in highmass MALDI-MS, equations used to determine the response in ESI-MS were adapted to our needs $[17,35]$. Here, we show the calculations for the case of $\mathrm{MBP}_{3}$ and MBP. The procedure for other samples is analogous.

In general, a response factor $(\mathrm{R})$ is defined as the ratio of the measured signal intensity (I) to the quantity (e.g., concentration) measured:

$$
\begin{aligned}
& \mathrm{I}_{\mathrm{MBP}}=[\mathrm{MBP}] \cdot \mathrm{R}_{\mathrm{MBP}} \\
& \mathrm{I}_{\mathrm{MBP}_{3}}=\left[\mathrm{MBP}_{3}\right] \cdot \mathrm{R}_{\mathrm{MBP}_{3}}
\end{aligned}
$$

The relative response is calculated as the ratio of two response factors. In this work, $\mathrm{R}_{\text {rel }}$ was always calculated as the ratio of the response of the substance with the higher molecular weight to the response of the substance with the lower molecular weight.

$\mathrm{R}_{\mathrm{rel}}=\frac{\mathrm{R}_{\mathrm{MBP}_{3}}}{\mathrm{R}_{\mathrm{MBP}}}$ 
Evaluation of the mass spectra measured allowed the calculation of the observed response factor $\left(\mathrm{R}_{\mathrm{obs}}\right)$, which is the ratio of the observed peak ratio.

$\mathrm{R}_{\mathrm{obs}}=\frac{\mathrm{I}_{\mathrm{MBP}_{3}}}{\mathrm{I}_{\mathrm{MBP}}}$

To take the molar ratio of the samples into account, the Equations $1 \mathrm{a}$ and $1 \mathrm{~b}$ are inserted into Equation 2, thus allowing the determination of $\mathrm{R}_{\mathrm{rel}}$ based on the peak ratios observed.

$\mathrm{R}_{\mathrm{rel}}=\frac{\mathrm{I}_{\mathrm{MBP}_{3}}}{\mathrm{I}_{\mathrm{MBP}}} \cdot \frac{[\mathrm{MBP}]}{\left[\mathrm{MBP}_{3}\right]}$

In Equations 3 and 4, $\mathrm{I}_{\mathrm{MBP}}$ and $\mathrm{I}_{\mathrm{MBP}_{3}}$ are the sums of all peak integrals that contained $\mathrm{MBP}$ or $\mathrm{MBP}_{3}$, respectively. In the case of MBP, a nonspecific dimer, $(\mathrm{MBP} \cdot \mathrm{MBP})^{+}$, is detected, the intensity of which needs to be taken into account twice. The same holds true for the nonspecific $\left(\mathrm{MBP} \cdot \mathrm{MBP}_{3}\right)^{+}$ cluster, which contributes to both $\mathrm{I}_{\mathrm{MBP}}$ and $\mathrm{I}_{\mathrm{MBP}_{3}}$.

$\mathrm{I}_{\mathrm{MBP}}=\mathrm{I}_{\mathrm{MBP}^{2+}}+\mathrm{I}_{\mathrm{MBP}^{+}}+2 \cdot \mathrm{I}_{(\mathrm{MBP} \cdot \mathrm{MBP})^{+}}+\mathrm{I}_{\left(\mathrm{MBP} \cdot \mathrm{MBP}_{3}\right)^{+}}$

$\mathrm{I}_{\mathrm{MBP}_{3}}=\mathrm{I}_{\mathrm{MBP}_{3}^{2+}}+\mathrm{I}_{\mathrm{MBP}_{3}^{+}}+\mathrm{I}_{\left(\mathrm{MBP}_{3} \cdot \mathrm{MBP}_{3}\right)^{+}}$

Following from the equations above, $\mathrm{R}_{\text {rel }}$ must be 1 if mass discrimination is absent. $\mathrm{R}_{\mathrm{rel}}>1$ means that the signals of the heavier ions, here $\mathrm{MBP}_{3}$, are more prominent than the ones from the lighter ones, here MBP. Analogously, $\mathrm{R}_{\text {rel }}<1$ indicates that the heavier ion is discriminated against.

\section{Results and Discussion}

Before the $\mathrm{R}_{\text {rel }}$ can be determined, it is important to investigate if the signals of the different species can be clearly distinguished or if some of the signals overlap because of similar $\mathrm{m} / \mathrm{z}$ ratios. As can be seen in Figure 1a and b, the MBP and $\mathrm{MBP}_{3}$ samples used were very pure. The measurement conditions were optimized such that only few multiply charged ions and few nonspecific clusters are detected. In the case of MBP (Figure 1a), doubly charged ions and a small signal of nonspecific (MBP-MBP) ${ }^{+}$clusters is detected. For $\mathrm{MBP}_{3}$, (Figure 1b) no aggregation was found at all, but a negligible amount $(<1.5 \%)$ of $\mathrm{MBP}_{3}{ }^{3+}$, which overlaps with $\mathrm{MBP}^{+}$. This was neglected in the analysis. Mixing both species in an equimolar ratio allowed measuring spectra such as the one shown in Figure 1c. All signals could be clearly assigned to the corresponding species, including a small signal of nonspecific $\left(\mathrm{MBP} \cdot \mathrm{MBP}_{3}\right)^{+}$clusters.

Since one of the most fundamental parameters in MALDI is laser pulse energy, the relative response factor as a function of the laser power was determined (Figure 2). The laser power
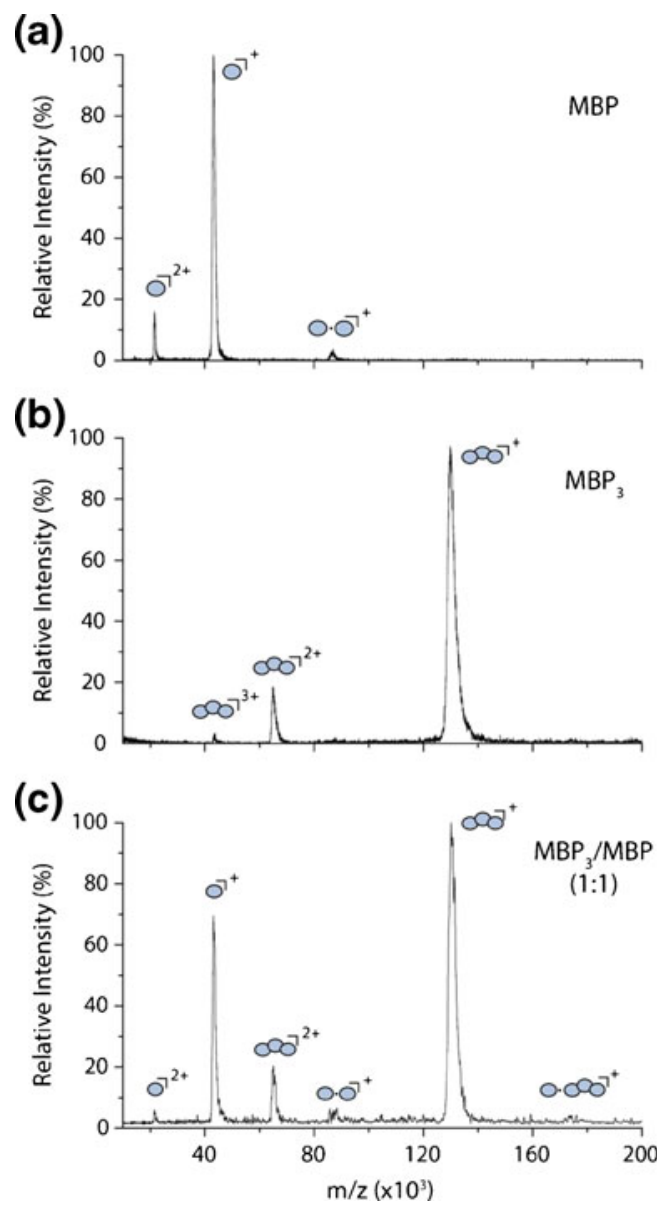

Figure 1. The spectra of $\mathrm{MBP}$ and $\mathrm{MBP}_{3}$ are shown in subfigures (a) and (b), respectively. As can be seen, only very little nonspecific aggregates and multiply charged ions are formed. Therefore, when measuring equimolar mixtures (c), no superposition of signals is observed and the individual peaks can be clearly assigned. To determine the relative response, the signal integrals were compared

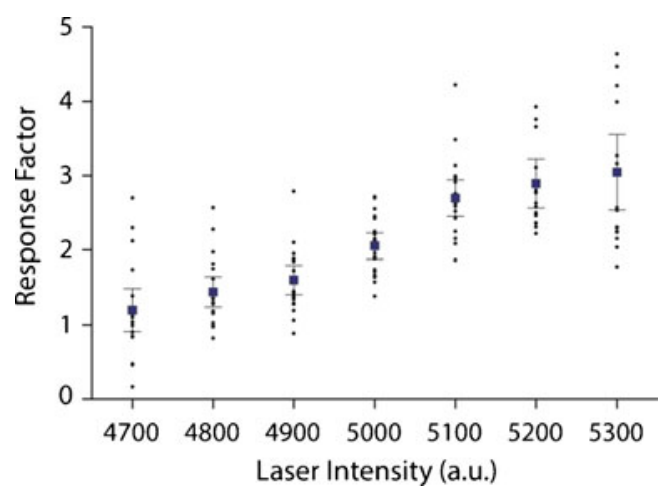

Figure 2. The influence of the laser power on the response factor is investigated by measuring several spots of an equimolar mixture of $\mathrm{MBP}_{3}$ and $\mathrm{MBP}$ at different laser intensities. The relative response of each measurement is calculated and plotted as well as the mean and the error, calculated using t-distribution $(95 \% \mathrm{Cl}$ ) of each measurement series 
range covered started with the ion formation threshold (in this case 4700 arbitrary units) and was increased in steps of 100 a.u. If no bias in ion transmission and detection is present, $\mathrm{R}_{\text {rel }}$ would be 1, as defined in Equation 2. However, the observed trend shows a clear tendency towards increased signal intensities of the heavier ion with increasing laser power. This is most probably because with higher laser power, the desorption step of the MALDI-process is facilitated for analytes with high masses [36, 37]. Once the analytes are ablated, the heavier ones might be easier to ionize since they possess a higher surface area, but otherwise the same sample properties as the analytes with lower masses. This correlation has been observed in previous work, $[5,19,38,39]$ but has, to our knowledge, never been studied systematically.

Therefore, to ensure that the response observed in the mass spectra represents the composition of the sample as closely as possible, it is important to perform MALDI near the ion formation threshold, where all constituents of the sample are ionized. The influence of laser power on the result of mass spectrometric measurements has already been investigated (i.e., in terms or resolution [40] and signal intensity $[36,41])$. For all the factors mentioned before, the optimal laser power condition was found to be slightly above the ion formation threshold. Therefore, when a typical MALDI-MS experiment is performed and the laser power is chosen accordingly, the measured protein ratio reflects the true ratio as closely as possible.

To investigate if mass discrimination is influenced by the stoichiometry of the analytes, the molar ratio of $\mathrm{MBP}_{3}$ and $\mathrm{MBP}$ was varied from 1:10 to 10:1; the total protein concentration was kept constant. The relative response factor as a function of the composition of the sample is depicted in Figure 3. If mass discrimination is absent, $R_{\text {rel }}$ must be 1 since it is corrected for the protein concentrations used (see Equation 2). $\mathrm{R}_{\text {rel }}>1$ means that the $\mathrm{MBP}_{3}$ signals are more prominent than the ones from

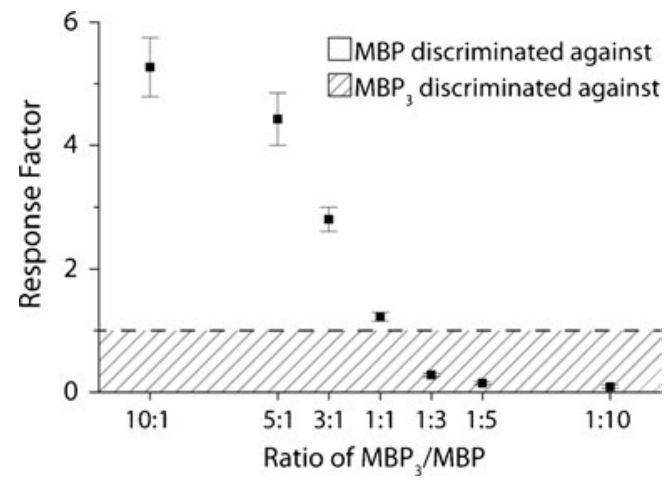

Figure 3. The ratio of $\mathrm{MBP}_{3}$ and $\mathrm{MBP}$ shows dramatic effects on the relative response factor. The more abundant species suppresses the less abundant one in a non-linear fashion. The error bars reflect absolute errors; therefore, they are more prominent for higher $R_{\text {rel }}$. If mass discrimination were absent, $R_{\text {rel }}$ would be 1 (dashed line), $R_{\text {rel }}<1$ (hatched segment) means that $\mathrm{MBP}_{3}$, and $R_{\text {rel }}>1$ that $M B P$ is discriminated against, respectively
MBP; $\mathrm{R}_{\text {rel }}<1$ corresponds to a suppression of $\mathrm{MBP}_{3}$ by MBP. $\mathrm{R}_{\text {rel }}$ for an equimolar ratio was found to be 1.22 , but upon changing the ratio of the two proteins we found that the species in molar excess always suppressed the less abundant one in a nonlinear fashion. We observed an $\mathrm{R}_{\text {rel }}$ of 5.27 for a ten-fold excess of $\mathrm{MBP}_{3}$, the ratio of the observed signals $\left(\mathrm{R}_{\text {obs }}\right)$ is $>52: 1$.

To understand if this suppression effect is mainly dependent on the sample's mass or on the properties of the sample itself as well, a similar experiment using different modular proteins was performed. To account for the nature of the sample, mixtures of the DARPin N8C and MBP were measured. As shown in Table 1, the molecular weights of the two proteins differ by only $7.3 \mathrm{kDa}$. The isoelectric point (pI) and the limit of detection (LoD) are similar as well. The samples could easily be detected as individual peaks (Figure $4 \mathrm{c}$ ) and $\mathrm{R}_{\text {rel }}$ was calculated (see Figure 4a). As in the previous experiment, the more concentrated species suppressed the other one, although the effect was much more prominent when two different species were compared: for a 10-fold molar excess of MBP over $\mathrm{N} 8 \mathrm{C}, \mathrm{R}_{\text {rel }}$ was determined as 42.15 . When the ratio MBP:N8C was $1: 10, R_{\text {rel }}$ was 0.08 , which is quite comparable to the value of 0.09 in case of $\mathrm{MBP}_{3}: \mathrm{MBP}(1: 10)$. However, when an equimolar mixture was investigated, the $\mathrm{R}_{\text {rel }}$ was also quite close to unity (0.89) but, in contrast to the previous experiment, the analyte with the lower mass (N8C) yielded a higher response, thus indicating that $\mathrm{N} 8 \mathrm{C}$ is easier to ionize than MBP.

To verify that this DARPin is easier to ionize than MBP (due to the sample's properties rather than due to the slight mass difference) MBP was exchanged by N10C, a DARPin with a molecular weight of $42.9 \mathrm{kDa}$. Using a mixture of N10C and $\mathrm{N} 8 \mathrm{C}$, it was possible to prove that the observed effect is due to the different ionization efficiencies. Determination of $\mathrm{R}_{\mathrm{rel}}$ of various $\mathrm{N} 10 \mathrm{C} / \mathrm{N} 8 \mathrm{C}$ mixtures showed a very similar trend (Figure 4b) as in the case of $\mathrm{MBP}_{3} / \mathrm{MBP}$ : when the two samples are similar in terms of their structure and composition, the suppression of the less concentrated species by the higher one is much less pronounced, but still visible. The two extreme scenarios when DARPins are used (1:10 and 10:1) result in $\mathrm{R}_{\text {rel }}$ of 8.05 and 0.18 , respectively. The equimolar mixture of $\mathrm{N} 10 \mathrm{C} / \mathrm{N} 8 \mathrm{C}$ also gave a similar result as $\mathrm{MBP}_{3} / \mathrm{MBP}: 1.12$ compared with 1.22 , respectively; here again, a slight preference for the heavier analyte is evident, although much less pronounced. The smaller effect can be explained by the smaller mass difference ( $7 \mathrm{kDa}$ compared with $86.7 \mathrm{kDa})$.

When only the equimolar mixtures within the same sample family were investigated, the suppression observed cannot originate from an excess of one species. Nevertheless, an $R_{\text {rel }} \neq 1$ is observed (see Table 2).

Summarizing the above results leads to the conclusion that the ionization probability does not correlate to the number of basic residues (pI) or the sensitivity of the instrument to the sample (LoD). Probably the solvent accessible surface influences the ability of the protein to carry a charge and therefore the $\mathrm{R}_{\text {rel }}$. This has been reported previously by Kaltashov and Mohimen for protein samples analyzed by ESI, where the 
(a)
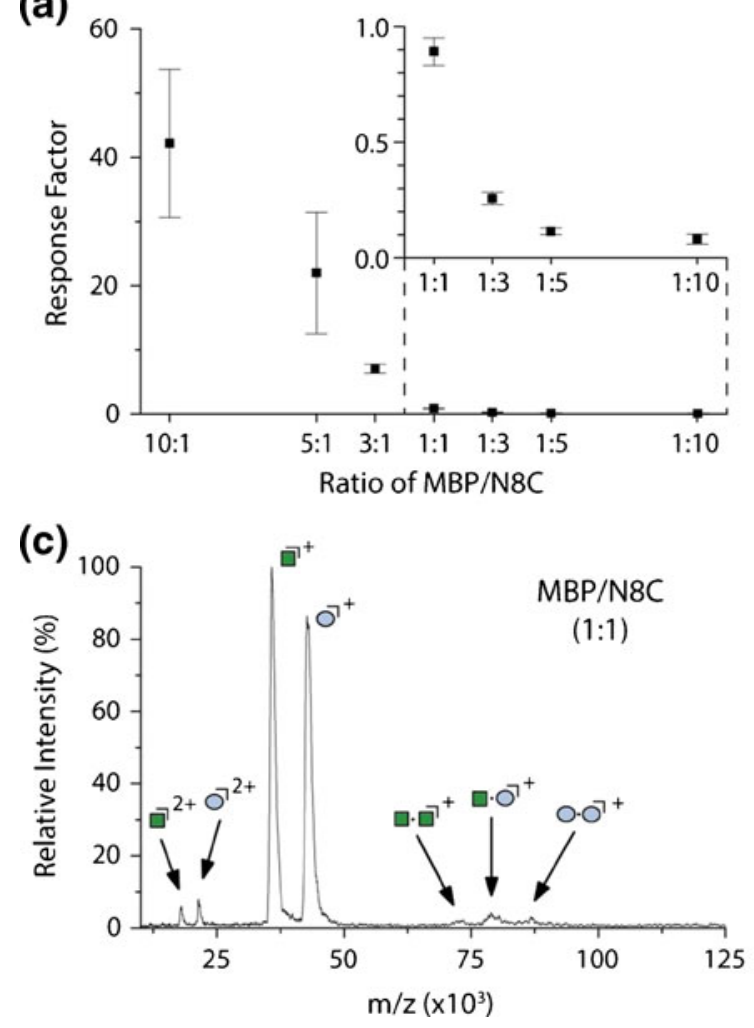

(b)

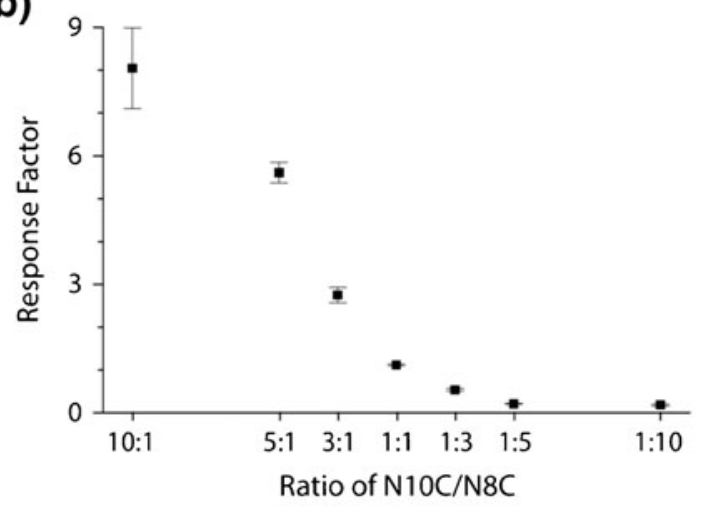

(d)

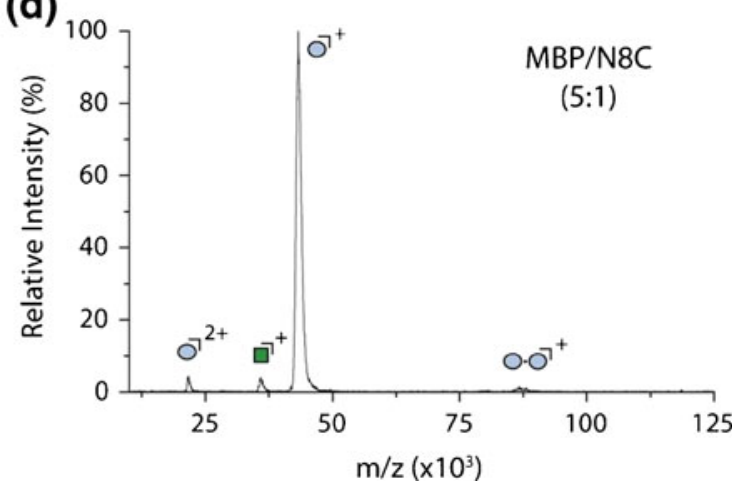

Figure 4. To investigate the influence of the sample on the relative response, $\mathrm{MPB}_{3}$ was substituted with a DARPin (N8C) of similar mass as MBP (a). Here, also the more abundant species suppresses the other species but the effect is about a factor of 10 more prominent. This is most probably due to different ionization properties of the two compounds. In contrast, mixtures of $\mathrm{N} 10 \mathrm{C}$ and $\mathrm{N} 8 \mathrm{C}$ showed a suppression behavior very similar to $\mathrm{MBP}_{3}$ and $\mathrm{MBP}(\mathbf{b})$. In (c) and (d), two mass spectra of different MBP/N8C mixtures are shown. The distinction between MBP (०) and N8C ( () is easily possible although the difference in molecular weights is only $4.1 \mathrm{kDa}$. The strong suppression of N8C by a 5 -fold excess of MBP is demonstrated in (d)

charge-state distribution of the sample, when sprayed under native conditions, was found to be independent on the number of acidic or basic residues but correlated with the accessible surface of the protein [42]. The samples investigated in this work have different shapes: MBP is a globular protein [43], whereas DARPins are more rod-like [33,34]. In recent work, a DARPin with high affinity towards MBP has been developed and a crystal structure has been solved [44]. Here, a direct visual comparison between MBP and a DARPin (however, not the one measured in this work) is possible. Following the argumentation of Kaltashov and Mohimen [42], it is clear that the samples with a higher surface, $\mathrm{MBP}_{3}$ and $\mathrm{N} 10 \mathrm{C}$, are ionized better than MBP and N8C, respectively. This slight preference might be overruled by the higher ionization probability of the

Table 2. Summary of the Observed Response Factors $\left(\mathrm{R}_{\mathrm{obs}}\right)$, the Relative Response Factors $\left(\mathrm{R}_{\mathrm{rel}}\right)$, Which are Corrected for the Concentration Ratio, and the Corresponding Errors, Calculated Using t-distribution (95\% CI)*

\begin{tabular}{|c|c|c|c|c|c|c|}
\hline \multirow[t]{2}{*}{ Molar Ratio } & \multicolumn{2}{|l|}{$\mathrm{MBP}_{3} / \mathrm{MBP}$} & \multicolumn{2}{|l|}{$\mathrm{MBP} / \mathrm{N} 8 \mathrm{C}$} & \multicolumn{2}{|l|}{ N10C/N8C } \\
\hline & $\mathrm{R}_{\mathrm{obs}}$ & $\mathrm{R}_{\text {rel }}$ & $\mathrm{R}_{\mathrm{obs}}$ & $\mathrm{R}_{\text {rel }}$ & $\mathrm{R}_{\mathrm{obs}}$ & $\mathrm{R}_{\text {rel }}$ \\
\hline $10: 1$ & $53 \pm 5$ & $5.27 \pm 0.48$ & $422 \pm 115$ & $42.15 \pm 11.51$ & $80 \pm 7$ & $8.05 \pm 0.94$ \\
\hline $5: 1$ & $22 \pm 2$ & $4.43 \pm 0.42$ & $110 \pm 47$ & $22.0 \pm 9.44$ & $28 \pm 1$ & $5.61 \pm 0.24$ \\
\hline $3: 1$ & $8.4 \pm 0.6$ & $2.80 \pm 0.20$ & $21 \pm 2$ & $7.07 \pm 0.70$ & $8.3 \pm 0.6$ & $2.75 \pm 0.18$ \\
\hline $1: 1$ & $1.22 \pm 0.07$ & $1.22 \pm 0.07$ & $0.89 \pm 0.06$ & $0.89 \pm 0.06$ & $1.12 \pm 0.02$ & $1.12 \pm 0.02$ \\
\hline $1: 3$ & $0.09 \pm 0.01$ & $0.28 \pm 0.03$ & $0.09 \pm 0.01$ & $0.26 \pm 0.03$ & $0.18 \pm 0.01$ & $0.54 \pm 0.03$ \\
\hline $1: 5$ & $0.029 \pm 0.005$ & $0.15 \pm 0.02$ & $0.023 \pm 0.003$ & $0.11 \pm 0.01$ & $0.040 \pm 0.002$ & $0.21 \pm 0.01$ \\
\hline $1: 10$ & $0.008 \pm 0.003$ & $0.09 \pm 0.03$ & $0.008 \pm 0.002$ & $0.08 \pm 0.02$ & $0.009 \pm 0.002$ & $0.18 \pm 0.02$ \\
\hline
\end{tabular}

*All values are calculated from more than 90 different measurements. The total protein concentration of $0.8 \mu \mathrm{M}$ was kept constant, but the molar ratio was varied. In general, it can be seen that the more prominent species suppresses the less prominent one. If different samples, such as an MBP polyprotein and a DARPin, are mixed, the different ionization properties show a more pronounced effect on $\mathrm{R}_{\mathrm{rel}}$ as when proteins of the same class are compared. 
DARPins compared with the MBP samples, thus an $\mathrm{R}_{\text {rel }}<1$ was found for the DARPin/MBP sample. Similarly to ESI, in MALDI ionization efficiencies of the samples might not be correlated to the number of basic residues.

Previous studies showed that DARPins are highly stable even when treated with heat [33] or denaturants [45]. MBP, on the other hand, forms a molten globule at low $\mathrm{pH}$ [46]. Even if the proteins are not in a native conformation anymore after MALDI-MS sample preparation, they should still maintain an overall rod-like or a globular shape, respectively.

Much more important for a good overall ion yield is the co-crystallization of the sample and the matrix molecules. It has been observed by Mädler et al. that even in negative mode MALDI-MS using an acidic matrix (SA) and adding TFA, good quality spectra from basic proteins could be obtained [47]. Different proteins do not co-crystallize equally well with the matrix used. Protein mixtures with different molar ratios of proteins might, therefore, crystallize inhomogeneously, and differences in the ionization efficiency of the samples are expected.

However, another reason for mass discrimination probably lies in the nature of the detector used. The HM2tuvo is based on ion-to-secondary ion conversion [28]. To the first and the second dynode, high voltages (typically $-20 \mathrm{kV}$ and $-2.5 \mathrm{kV}$, respectively) are applied. Ions impinging on the first dynode sputter secondary ions that are accelerated between the two dynodes by the applied potential difference. Only on the second dynode, electrons are generated, multiplied, and detected. The yield of secondary ions depends on the mass of the impinging ion. This phenomenon is known from secondary ion mass spectrometry, where the use of heavy ion clusters instead of light single-primary ions increases the secondary ion yield after impact [29, 30]. Similarly, with ICDs, high-mass ions create more secondary ions and, subsequently, more electrons. The signal of heavy molecules detected in mass spectrometry might thus be overestimated systematically when using ICD-based detectors. Depending on the ionization probability of the samples investigated, this preference due to the detector efficiency might be overruled by a preference in ion formation. However, compared with the results of Farmer and Caprioli, who used an MCP [19], our results show a much reduced mass discrimination effect. In their studies, they found that a correction factor of 4.4 that was necessary to compensate for mass discrimination of a peak at $\mathrm{m} / \mathrm{z}=150,000$ Th relative to one at 50,000 Th. To calculate a correction factor, one can simply calculate $1 / \mathrm{R}_{\text {rel }}$. For the setup used here and an equimolar mixture of $\mathrm{MBP}_{3}$ and $\mathrm{MBP}, \mathrm{R}_{\text {rel }}$ was measured as 1.22 ; thus, the correction factor would be 0.82 for peaks measured at $m / z=130,000 \mathrm{Th}\left(\mathrm{MBP}_{3}\right)$ and 43,000 Th (MBP), respectively. They also found that the peak area ratio did not change with laser power. Probably, when using an MCP detector, the reduced sensitivity for higher masses and the enhanced ion production of low-mass ions cancel out. The use of an ICD-based detector results in a clear preference for higher masses at higher laser power. Whether this phenomenon is linear or whether a region of highest sensitivity exists, will need further investigation in the future. A better understanding of the processes happening in the ICD is crucial for using high-mass MALDI-MS to monitor the composition of complex samples with high precision.

As shown above, the relative response strongly depends on different parameters such as the laser power chosen, sample composition and the properties of the sample constituents. As opposed to the laser power, the sample composition and the sample constituents can usually not be freely chosen. As long as the composition is approximately equimolar, the relative response factor will be close to unity. Unfortunately, the more the composition deviates from an equimolar ratio, the stronger the discrimination effect. In an extreme case, it can lead to complete suppression of one of the compounds.

The results found here are of importance for any application of high-mass MALDI-MS. Although mass discrimination exists not only when using MCPs but also with ICDs, the discrimination is reduced a lot. Knowing this allows taking certain precautions to minimize the extent of mass discrimination. When the experiment is planned carefully, the discrimination might be reduced to a level allowing neglecting it.

\section{Conclusions}

The relative response factors of protein oligomers and DARPins with different masses and at different molar ratios were determined for MALDI-MS experiments. As detector, an ICD specifically designed for detection of high-mass molecules was used. We found that the relative response is influenced by the laser power, the sample composition, and the ionization efficiency of the sample constituents.

It was not possible to unify all effects found into one correction factor. Nonetheless, some general guidelines that might be followed for planning an experiment are presented: (1) use of a laser power just above the threshold for ion formation gives the most accurate relative response factor, (2) if possible, the sample constituents should be as similar as possible to each other to ensure similar ionization properties, and (3) great excess of one component over the other should be avoided. Although it might not be possible to follow these guidelines in every case (for example, the analyte usually cannot be changed), they help to reduce the mass discrimination to a great extent and allow high-mass MALDI-MS measurements that reflect the sample composition better.

\section{Acknowledgments}

The authors thank the SystemsX.ch initiative (CINA grant) and the Swiss National Science Foundation (grant no. 200020124663) for their financial support. G.M. was supported by a Swiss Government Excellence Scholarship (2011-2013). The authors also thank Stefanie Mädler (currently at York University, Toronto, Canada) and Nadezhda Nespovitaya (ETH Zürich, Switzerland) for their help with the design and synthesis of the $\mathrm{MBP}_{3}$ polyproteins, and the Plückthun Lab 
(University of Zürich, Switzerland) for the generous gift of the DARPin samples.

\section{References}

1. Beavis, R.C., Chait, B.T.: Rapid, sensitive analysis of protein mixtures by mass-spectrometry. Proc. Natl. Acad. Sci. U.S.A. 87(17), 6873-6877 (1990)

2. Bahr, U., Deppe, A., Karas, M., Hillenkamp, F., Giessmann, U.: Mass spectrometry of synthetic polymers by UV-matrix-assisted laser desorption/ionization. Anal. Chem. 64(22), 2866-2869 (1992)

3. Montaudo, G., Montaudo, M.S., Puglisi, C., Samperi, F.: Characterization of polymers by matrix-assisted laser desorption/ionization time-of-flight mass spectrometry: Molecular weight estimates in samples of varying polydispersity. Rapid Commun. Mass Spectrom. 9(5), 453-460 (1995)

4. Montaudo, G., Montaudo, M.S., Puglisi, C., Samperi, F.: Characterization of polymers by matrix-assisted laser-desorption ionization-time of flight mass-spectrometry. End group determination and molecular-weight estimates in poly(ethylene glycols). Macromolecules 28(13), 4562-4569 (1995)

5. Martin, K., Spickermann, J., Räder, H.J., Müllen, K.: Why does matrixassisted laser desorption/ionization time-of-flight mass spectrometry give incorrect results for broad polymer distributions? Rapid Commun. Mass Spectrom. 10(12), 1471-1474 (1996)

6. Schriemer, D.C., Li, L.: Mass discrimination in the analysis of polydisperse polymers by MALDI time-of-flight mass spectrometry. 1 . Sample preparation and desorption/ionization issues. Anal. Chem. 69(20), 4169-4175 (1997)

7. Schriemer, D.C., Li, L.: Mass discrimination in the analysis of polydisperse polymers by MALDI time-of-flight mass spectrometry. 2 . Instrumental issues. Anal. Chem. 69(20), 4176-4183 (1997)

8. Shimada, K., Lusenkova, M.A., Sato, K., Saito, T., Matsuyama, S., Nakahara, H., Kinugasa, S.: Evaluation of mass discrimination effects in the quantitative analysis of polydisperse polymers by matrix-assisted laser desorption/ionization time-of-flight mass spectrometry using uniform oligostyrenes. Rapid Commun. Mass Spectrom. 15(4), 277$282(2001)$

9. Shimada, K., Nagahata, R., Kawabata, S.-I., Matsuyama, S., Saito, T., Kinugasa, S.: Evaluation of the quantitativeness of matrix-assisted laser desorption/ionization time-of-flight mass spectrometry using an equimolar mixture of uniform poly(ethylene glycol) oligomers. J. Mass Spectrom. 38(9), 948-954 (2003)

10. Pieles, U., Zürcher, W., Schär, M., Moser, H.E.: Matrix-assisted laser desorption ionization time-of-flight mass-spectrometry: A powerful tool for the mass and sequence analysis of natural and modified oligonucleotides. Nucleic Acids Res. 21(14), 3191-3196 (1993)

11. Roskey, M.T., Juhasz, P., Smirnov, I.P., Takach, E.J., Martin, S.A., Haff, L.A.: DNA sequencing by delayed extraction matrix-assisted laser desorption/ionization time of flight mass spectrometry. Proc. Natl. Acad. Sci. U.S.A. 93(10), 4724-4729 (1996)

12. Shaler, T.A., Tan, Y., Wickham, J.N., Wu, K.J., Becker, C.H.: Analysis of enzymatic DNA-sequencing reactions by matrix-assisted laserdesorption ionization time-of-flight mass-spectrometry. Rapid Commun. Mass Spectrom. 9(10), 942-947 (1995)

13. Chen, X., Westphall, M.S., Smith, L.M.: Mass spectrometric analysis of DNA mixtures: Instrumental effects responsible for decreased sensitivity with increasing mass. Anal. Chem. 75(21), 5944-5952 (2003)

14. Farmer, T.B., Caprioli, R.M.: Assessing the multimeric states of proteins: Studies using laser desorption mass spectrometry. Biol. Mass Spectrom. 20(12), 796-800 (1991)

15. Farmer, T.B., Caprioli, R.M.: Determination of protein-protein interactions by matrix-assisted laser desorption/ionization mass spectrometry. J. Mass Spectrom. 33(8), 697-704 (1998)

16. Mädler, S., Seitz, M., Robinson, J., Zenobi, R.: Does chemical crosslinking with NHS esters reflect the chemical equilibrium of proteinprotein noncovalent interactions in solution? J. Am. Soc. Mass Spectrom. 21(10), 1775-1783 (2010)

17. Gabelica, V., Galic, N., Rosu, F., Houssier, C., De Pauw, E.: Influence of response factors on determining equilibrium association constants of noncovalent complexes by electrospray ionization mass spectrometry. $J$. Mass Spectrom. 38(5), 491-501 (2003)
18. Weidmann, S., Barylyuk, K., Nespovitaya, N., Mädler, S., Zenobi, R.: A new, modular mass calibrant for high-mass MALDI-MS. Anal. Chem. 85(6), 3425-3432 (2013)

19. Farmer, T.B., Caprioli, R.M.: Mass discrimination in matrix-assisted laser-desorption ionization time-of-flight mass-spectrometry: A study using cross-linked oligomeric complexes. J. Mass Spectrom. 30(9), 1245-1254 (1995)

20. Wiza, J.L.: Microchannel plate detectors. Nucl. Inst. Methods 162(1-3), 587-601 (1979)

21. Beuhler, R.J., Friedman, L.: Threshold studies of secondary-electron emission induced by macro-ion impact on solid-surfaces. Nucl. Inst. Methods 170(1-3), 309-315 (1980)

22. Geno, P.W., Macfarlane, R.D.: Secondary electron emission induced by impact of low-velocity molecular ions on a microchannel plate. Int. J. Mass Spectrom. Ion Process. 92, 195-210 (1989)

23. Brunelle, A., Chaurand, P., Della-Negra, S., Le Beyec, Y., Baptista, G.B.: Surface secondary-electron and secondary-ion emission induced by large molecular ion impacts. Int. J. Mass Spectrom. Ion Process. 126, 65-73 (1993)

24. Coeck, S., Beck, M., Delauré, B., Golovko, V.V., Herbane, M., Lindroth, A., Kopecky, S., Kozlov, V.Y., Kraev, I.S., Phalet, T., Severijns, N.: Microchannel plate response to high-intensity ion bunches. Nucl. Inst. Methods A 557(2), 516-522 (2006)

25. Frank, M., Labov, S.E., Westmacott, G., Benner, W.H.: Energysensitive cryogenic detectors for high-mass biomolecule mass spectrometry. Mass Spectrom. Rev. 18(3-4), 155-186 (1999)

26. Kraus, H.: Cryogenic detectors and their application to mass spectrometry. Int. J. Mass Spectrom. 215(1-3), 45-58 (2002)

27. Park, J., Qin, H., Scalf, M., Hilger, R.T., Westphall, M.S., Smith, L.M., Blick, R.H.: A mechanical nanomembrane detector for time-of-flight mass spectrometry. Nano Lett. 11(9), 3681-3684 (2011)

28. Wenzel, R.J., Röhling, U., Nazabal, A., Hillenkamp, F.: A detector device for high mass ion detection, a method for analyzing ions of high mass and a device for selection between ion detectors. Internat. Patent WO2009086642-A1, July 16 (2009).

29. Winograd, N.: The magic of cluster SIMS. Anal. Chem. 77(7), 142 A149 A (2005)

30. Fletcher, J.S., Lockyer, N.P., Vickerman, J.C.: Developments in molecular SIMS depth profiling and 3D imaging of biological systems using polyatomic primary ions. Mass Spectrom. Rev. 30(1), 142-174 (2011)

31. Chen, F., Gerber, S., Heuser, K., Korkhov, V.M., Lizak, C., Mireku, S., Locher, K.P., Zenobi, R.: High-mass matrix-assisted laser desorption ionization-mass spectrometry of integral membrane proteins and their complexes. Anal. Chem. 85(7), 3483-3488 (2013)

32. Wetzel, S.K., Settanni, G., Kenig, M., Binz, H.K., Plückthun, A.: Folding and unfolding mechanism of highly stable full-consensus ankyrin repeat proteins. J. Mol. Biol. 376(1), 241-257 (2008)

33. Binz, H.K., Stumpp, M.T., Forrer, P., Amstutz, P., Plückthun, A.: Designing repeat proteins: Well-expressed, soluble and stable proteins from combinatorial libraries of consensus ankyrin repeat proteins. $J$. Mol. Biol. 332(2), 489-503 (2003)

34. Forrer, P., Binz, H.K., Stumpp, M.T., Plückthun, A.: Consensus design of repeat proteins. ChemBioChem 5(2), 183-189 (2004)

35. Boeri Erba, E., Barylyuk, K., Yang, Y., Zenobi, R.: Quantifying protein-protein interactions within noncovalent complexes using electrospray ionization mass spectrometry. Anal. Chem. 83(24), 92519259 (2011)

36. Dreisewerd, K.: The desorption process in MALDI. Chem. Rev. 103(2), 395-425 (2003)

37. Knochenmuss, R.: Ion formation mechanisms in UV-MALDI. Analyst 131(9), 966-986 (2006)

38. Aksenov, A.A., Bier, M.E.: The analysis of polystyrene and polystyrene aggregates into the mega dalton mass range by cryodetection MALDI TOF MS. J. Am. Soc. Mass Spectrom. 19(2), 219-230 (2008)

39. Zenobi, R.: Ionization processes and detection in MALDI-MS of polymers. In: Li, L. (ed.) MALDI Mass Spectrometry for Synthetic Polymer Analysis, pp. 9-26. John Wiley and Sons, Inc, Hoboken, NJ (2009)

40. Ingendoh, A., Karas, M., Hillenkamp, F., Giessmann, U.: Factors affecting the resolution in matrix-assisted laser desorption-ionization mass spectrometry. Int. J. Mass Spectrom. Ion Process. 131, 345-354 (1994)

41. Knochenmuss, R., Zenobi, R.: MALDI ionization: The role of in-plume processes. Chem. Rev. 103(2), 441-452 (2003) 
42. Kaltashov, I.A., Mohimen, A.: Estimates of protein surface areas in solution by electrospray ionization mass spectrometry. Anal. Chem. 77(16), 5370-5379 (2005)

43. Spurlino, J.C., Lu, G.-Y., Quiocho, F.A.: The 2.3-Å resolution structure of the maltose- or maltodextrin-binding protein, a primary receptor of bacterial active transport and chemotaxis. J. Biol. Chem. 266(8), 5202-5219 (1991)

44. Binz, H.K., Amstutz, P., Kohl, A., Stumpp, M.T., Briand, C., Forrer, P., Grütter, M.G., Plückthun, A.: High-affinity binders selected from designed ankyrin repeat protein libraries. Nat. Biotechnol. 22(5), 575582 (2004)
45. Kohl, A., Binz, H.K., Forrer, P., Stumpp, M.T., Plückthun, A., Grütter, M.G.: Designed to be stable: Crystal structure of a consensus ankyrin repeat protein. Proc. Natl. Acad. Sci. U.S.A. 100(4), 1700-1705 (2003)

46. Prajapati, R.S., Indu, S., Varadarajan, R.: Identification and thermodynamic characterization of molten globule states of periplasmic binding proteins. Biochemistry 46(36), 10339-10352 (2007)

47. Mädler, S., Barylyuk, K., Boeri Erba, E., Nieckarz, R.J., Zenobi, R.: Compelling advantages of negative ion mode detection in high-mass MALDI-MS for homomeric protein complexes. J. Am. Soc. Mass Spectrom. 23(2), 213-224 (2012) 